\title{
Testing the Plant Growth-Defense Hypothesis Belowground: Do Faster-Growing Herbaceous Plant Species Suffer More Negative Effects from Soil Biota than Slower-Growing Ones?
}

\author{
Stefanie Lemmermeyer, Linda Lörcher, Mark van Kleunen, and Wayne Dawson \\ Biology Department, University of Konstanz, Universitätsstrasse 10, Konstanz D-78457, Germany \\ Submitted June 4, 2014; Accepted March 17, 2015; Electronically published May 18, 2015 \\ Online enhancements: appendixes. Dryad data: http://dx.doi.org/10.5061/dryad.49s82.
}

\begin{abstract}
According to the growth-defense hypothesis in ecology, faster-growing plant species should suffer more from herbivores and pathogens than slower-growing species. Tests of this hypothesis have focused on aboveground plant tissues, herbivores, and pathogens; however, it should also apply to root defense. To test whether fastergrowing species suffer more negatively from soil biota than slowergrowing species, we estimated first-season growth rates of 34 herbaceous plant species and used weighted linear regressions to assess the relationship between growth rates and responses to being grown in sterilized versus unsterilized soil (biotic soil effects) and to growing in soil previously occupied by conspecifics versus a mixture of species (conspecific soil effects). We found a negative relationship between relative growth rate and biotic soil effects, with slower-growing species tending to suffer less or even benefit from the presence of soil biota, while faster-growing species were more negatively affected. Biotic soil effects were also negatively related to size-corrected growth rates. These relationships remained negative after accounting for influential species, but a large amount of variation remained unexplained. Moreover, there was no clear relationship between growth rates and conspecific soil effects. A simple relationship between growth and defense aboveground may not be so clearly reflected belowground because of the many interacting antagonistic and mutualistic organisms likely involved.
\end{abstract}

Keywords: life history, natural enemies, plant-soil feedback, soil microbes.

\section{Introduction}

The hypothesized relationship between the ability of plants to grow fast and the ability to defend themselves against natural enemies is a fundamental concept in ecology (Coley et al. 1985; Coley 1988; Herms and Mattson 1992; Van Zandt 2007; Kempel et al. 2011). Recent global-scale (Lind et al. 2013) and meta-analytical (Endara and Coley 2011)

* Corresponding author; e-mail: wayne.dawson@uni-konstanz.de.

Am. Nat. 2015. Vol. 186, pp. 264-271. (C) 2015 by The University of Chicago. 0003-0147/2015/18602-55522\$15.00. All rights reserved.

DOI: $10.1086 / 682005$ studies suggest that a relationship between the ability of plants to grow fast and the ability to defend themselves against natural enemies is the norm among multiple species in different communities. Faster-growing species occupy resource-rich environments and invest resources in plant growth rather than defense against enemies. In contrast, slower-growing species, often from resource-poor environments, invest more in defenses, preventing losses of plant tissue at a cost to plant growth.

Although a growth-defense relationship appears to be the norm, most work to date has been focused aboveground (Coley et al. 1985; Coley 1988; Herms and Mattson 1992; Van Zandt 2007; Kempel et al. 2011). The role played by belowground natural enemies has received less attention (Rasmann et al. 2011) despite evidence that soil pathogens, particularly fungi, can cause high rates of root (Eissenstat and Yanai 1997) and seedling (Jarosz and Davelos 1995; Packer and Clay 2000) mortality. There is growing interest in potential regulation of individual- and population-level plant performance by density-dependent effects of soil biota (Mangan et al. 2010; van der Putten et al. 2013). The concept of plant-soil feedback suggests that, over time, species-specific pathogens accumulate in the soil occupied by individual plants, such that subsequent generations of individuals of the same species experience reduced growth and fitness (Bever 1994). The net soil biota effects are often negative, suggesting that species-specific fungal and bacterial pathogens outweigh more generalist mutualists (e.g., mycorrhizal fungi) in their effects on plant growth (Kulmatiski et al. 2008). Thus, plants should perform less well on soils previously occupied by conspecifics compared with those previously occupied by other species. However, the strength and direction of net soil biota effects vary extensively among species and studies (Kulmatiski et al. 2008).

Susceptibility to belowground natural enemies could depend on successional stage and growth rates (Rasmann 
et al. 2011). If a relationship between plant growth and defense occurs belowground, early successional and fastergrowing plant species should be more susceptible to root herbivores and pathogens than slower-growing species. Faster-growing species are thought to produce thinner roots that are less well defended physically or chemically than slower-growing species (Rasmann et al. 2011). However, a direct test of the association between growth rates or root traits and effects of soil microbial communities on plant growth is currently lacking. Specific root length (SRL) represents the length of root deployed for water and nutrient uptake per unit of mass invested. High SRL has been linked to high rates of root proliferation (Eissenstat 1991), greater branching intensity, and thinner roots (Comas and Eissenstat 2009). High root N content of plant tissue correlates with less dense roots and shorter root life span, and these traits should characterize faster-growing but less-well-defended species (Eissenstat and Yanai 1997; Tjoelker et al. 2005; Reich 2014). Slower-growing plant species are thought to better defend their roots with carbonbased lignins and phenolic compounds than faster-growing species (Eissenstat and Yanai 1997). We therefore expect SRL and $\mathrm{C}: \mathrm{N}$ ratio to be correlated with species growth rates.

In this study, we assessed the relationship between growth rates and the net effects of soil biota (microbial fungi and bacteria) on plant growth. We estimated first-season growth rates for 34 herbaceous plant species in central Europe using nonlinear growth curve models (Paine et al. 2012). We then measured the magnitude and direction of the effect of soil biota on these same species in a second experiment by growing the plants on soil previously conditioned by the same species (conspecific, which was either sterilized or unsterilized to measure biotic soil effects) or a mixture of species (heterospecific). We also independently measured SRL and root $\mathrm{C}: \mathrm{N}$ ratio as root traits that could influence the vulnerability of plant roots to pathogens. We predicted the following: (1) that plant species with faster growth experience more negative biotic soil effects, that faster-growing species grow less well on conspecific unsterilized soil than sterilized soil, and that slowergrowing species, in contrast, are less negatively affected by unsterilized than sterilized soil; (2) that faster-growing species also suffer more negatively than slower-growing species when growing in conspecific soils compared with soils from a mixture of species if species-specific soil pathogens accumulate in conspecific soils; (3) that root traits are correlated with growth rates, specifically that fastergrowing species have lower $\mathrm{C}: \mathrm{N}$ ratios and higher SRL than slower-growing species; and (4) that plants with a high SRL and a low $\mathrm{C}: \mathrm{N}$ ratio suffer more negatively from soil biota than species with a lower SRL and a higher C:N ratio.

\section{Material and Methods}

Study Species

We collected seeds from wild populations of 34 herbaceous, mostly perennial plant species in southern Germany and Switzerland during 2012 (see table A1; tables A1-A6 are available online). Species were selected to represent a broad range of growth rates based on Grime and Hunt (1975). If possible, we collected seeds from 10 parent plants per population; for two species we had six parent plants, and for one species we had three parent plants. The seeds were stored in cool, dry conditions until April 2013.

\section{Growth Rate Estimation}

For full details on growth rate estimation methods, see appendix B (apps. A-C are available online). In brief, we counted out 36 batches of 10 seeds per species from a mixture of 1,000 seeds with equal contributions from each parent plant. We sowed each batch of 10 seeds in 1.12-L pots filled with a mixture of topsoil, vermiculite, and washed sand (ratio of $1: 1: 1$ by volume) on April 22 and 23, 2013. The pots were checked every 2 days after sowing for germinated seeds until all or most pots per species had emergent seedlings, and this date per species was designated week 0 . After removing all but one of the germinated seedlings per pot, up to three plants (i.e., pots) were harvested weekly per species from weeks 1 to 12 (see app. B for calculation of week 0 seedling biomass). This gave a total of 36 plants for most species (see app. B for information on species with fewer plants harvested).

We estimated species growth rates in two ways. First, we calculated classical relative growth rate (RGR) as the difference in $\ln$ (mean biomass) between weeks 10 and 0 divided by time (70 days), giving an estimate of RGR in grams per gram per day. We chose 10 weeks instead of 12 because plants were harvested only until week 10 for one of the species, Lotus corniculatus. Because classical RGR inherently decreases with increasing plant size (Turnbull et al. 2008), we also estimated size-corrected growth rate (SGR) at a common plant size for all species by fitting nonlinear growth curve models for each species' biomass over all weeks for which data were available. We largely followed the protocol and used the R program scripts provided by Paine et al. (2012). Four types of growth function were fitted: monomolecular, three-parameter logistic, four-parameter logistic, and Gompertz. SGR was then estimated using the best-fitting model (identified as the model with the lowest Akaike information criterion [AIC]) at the average biomass of plants $(3.52 \mathrm{~g})$ calculated across all species in week 6 (the midpoint of the total growth period for most species). When the lowest AIC model 
was not distinguishable (i.e., difference of $<2$ AIC units; Burnham and Anderson 2002) from other models, an average SGR was calculated from the estimates of the models concerned (table A1). The SGR can be thought of as representing an average SGR equivalent to conventional RGR, which is also an average (Turnbull et al. 2012). The estimated RGR and SGR are shown in table A1. Our growth rate estimates reflect first-season growth from seed of the species. This life stage is relevant to consider in our study because seedlings and younger plants are likely to be susceptible to pathogen attack, while seedling growth and survival of mycorrhizal-dependent species would depend on successful mycorrhization of their roots (Kardol et al. 2013).

\section{Effects of Soil Biota}

For full details on how effects of soil biota were measured, see appendix C. During May 2013, we filled 170 pots (4.5 $\mathrm{L}$ in volume) with a substrate consisting of a mixture of sand, vermiculite, and topsoil (as used previously, with a ratio of $1: 1: 1$ ) to $4 \mathrm{~L}$ and then added an extra $200 \mathrm{~mL}$ of sieved, homogenized soil collected from the field to each pot, which was thoroughly mixed with the other substrate. The field-collected soil was obtained from seven meadow/ grassland areas in the vicinity of the University of Konstanz (see app. C and table A2 for collection details). Fieldcollected soil was used to inoculate the substrate with a larger range of soil biota for the conditioning phase. For each species, we then sowed 100 seeds (from the same source as the seeds used in growth rate estimation) in each of five replicate pots on May 22 and 23, 2013 (the pots were placed outside). Once the seeds had germinated, we removed excess seedlings until five remained (evenly spaced) in each pot. These plants were then grown for 14 weeks until September 17-18, 2013. After this soil-conditioning phase, the aboveground biomass per pot was removed, and the soil per individual pot was sieved to remove the majority of roots.

We then filled 510 pots ( $1.2 \mathrm{~L}$ in volume) with $1 \mathrm{~L}$ of $1: 1$ washed sand and vermiculite and $100 \mathrm{~mL}$ of soil from the conditioning phase that represented one of the following three treatments: (1) unsterilized soil from a replicate conditioning pot of one species (conspecific soil), (2) sterilized soil from a replicate conditioning pot of one species (conspecific, sterilized; soil was sterilized at $121^{\circ} \mathrm{C}$ for $40 \mathrm{~min}$ in an autoclave), and (3) unsterilized soil from a mixture of soils made from one replicate conditioning pot of every species (heterospecific soil). This mixture included soil occupied by every species in equal measure, including the planted target, and so represented a dilution of accumulated conspecific soil biota. This treatment is realistic, as microbes that affect plants and accumulate in their soils in a plant species-specific manner may not be completely absent from soil unoccupied by the host species (Maron et al. 2014).

Conspecific and heterospecific soil treatments were always paired according to the replicate conditioning pot used for the soil inocula. Because we had five replicate conditioning pots for each of the 34 species, we then had a maximum of five replicate $1.2-\mathrm{L}$ pots per species for each of the above-described soil inoculum treatments. This gave a total of 15 pots per species. In each pot per species we planted a single seedling, grown from seeds representing the same parent plants as those used during the conditioning phase and for growth rate estimation. The plants were then grown in a greenhouse for 12 weeks, watered weekly, and fertilized every 2 weeks with $100 \%$ Hoagland solution (see app. B for details on growing conditions and table A3 for the Hoagland solution recipe). After 12 weeks of growth, the aboveground biomass was harvested and dried at $70^{\circ} \mathrm{C}$ for $72 \mathrm{~h}$. The belowground biomass was washed carefully to remove the substrate before drying. Both belowground and aboveground biomasses were then weighed, and total biomass was calculated.

\section{SRL and Root $C: N$ Ratio}

Five replicate plants of each species were grown simultaneously with the plants used to measure soil biota effects for 10 weeks to estimate SRL and root C:N ratio (see app. B for details). After 10 weeks and immediately after washing, the plant root systems were stored in water for a maximum of $24 \mathrm{~h}$. Two subsamples were taken (blindly) from each replicate root system and stained using neutral red root-staining solution. An 8-bit greyscale image of each subsample was taken at 600 dpi with a flatbed scanner (Epson Expression 10000 XL; Regent Instruments, Quebec). Total root length per subsample was then measured using the WinRHIZO program (2012; Regent Instruments). SRL was then calculated per subsample by dividing root length $(\mathrm{cm})$ by root dry mass (after drying for $72 \mathrm{~h}$ at $70^{\circ} \mathrm{C}$ ). An average SRL from the two subsamples per plant was calculated, and average SRL for the species was calculated from the replicate plants.

To estimate root $\mathrm{C}: \mathrm{N}$ ratio, $\sim 3 \mathrm{mg}$ of dried (unstained) root biomass per plant was ground into a powder using a milling machine (MM 300; Retsch, Haan, Germany). The $\mathrm{C}$ and $\mathrm{N}$ content of $0.3-0.9 \mathrm{mg}$ of powdered root per plant was then measured using a CHNSO combustion analyzer (Euro EA; HEKAtech, Wegberg, Germany). The mean root $\mathrm{C}: \mathrm{N}$ ratio was then calculated per species. Lathyrus pratensis was excluded from the SRL and C:N ratio analysis and Hypericum perforatum and Geum urbanum were excluded from the $\mathrm{C}: \mathrm{N}$ analysis because of measurement problems and limited root material. 


\section{Analysis}

We calculated the biotic soil effect as the mean difference in biomass between plants growing in unsterilized and sterilized conspecific soil from the replicate pairs of plants grown. We also calculated the conspecific soil effect as the mean difference in biomass between plants grown in conspecific and heterospecific soil. The sterilization approach has been advocated for determining the strength and sign of general soil feedback effects, while the conspecificheterospecific approach has the advantage of avoiding unwanted effects of sterilization procedures (Brinkman et al. 2010). Because some plants died, soil effects were occasionally measured for fewer than five replicates per species (see table A1). We also calculated the variance of biotic soil effects and conspecific soil effects per species. Origanum vulgare was excluded from analyses of conspecific soil effects because of insufficient numbers of surviving plants.

To assess the relationships between species mean growth rates (RGR or SGR) or root traits and biotic or conspecific soil effects, we fitted weighted linear regression models using the function $\operatorname{lm}()$ in the program R. Each species' mean biotic or conspecific soil effect was weighted in the model by the reciprocal of the species' variance of the effect (added to the model using weights $=$ ). To account for variation in plant size among species, the mean summed biomass of plants in the replicate pairs used to calculate soil effects was included as a covariate (centered on the overall mean and scaled by the standard deviation) in analyses with RGR and root traits. This covariate was excluded from models considering SGR because SGR is already size corrected and there was collinearity between the two variables $(r=0.62)$. We then compared the fit of the models including RGR to that of the models including only the biomass covariate as well as the fit of the models including SGR to that of intercept models, using the AIC. If AICs for models including growth rates were $>2$ units smaller than AICs for the simpler comparator models, then the growth rate model was considered a better fit. When a model including growth rates or root traits gave a better fit than the simpler model, we inspected the model estimates. A relationship between SGR, RGR, SRL, or root C:N and soil effects could be driven by influential species in the data set. To address this, we removed each species, one at a time, and inspected changes in the parameter estimates (plus their direction) as a measure of each species' influence.

Because plant species have varying degrees of phylogenetic relatedness among them (Felsenstein 1985), we reran the regressions described above incorporating phylogenetic information using phylogenetic generalized least squares models with the $\mathrm{R}$ package nlme (Pinheiro et al. 2014). Trait-growth rate correlations were redone using phylogenetically independent contrasts (Felsenstein 1985).
We used a phylogenetic tree of the 34 species constructed using the online program Phylomatic (ver. 3; Webb and Donoghue 2005; http://phylodiversity.net/phylomatic/) and the Angiosperm Phylogeny Website (Chase and Reveal 2009; http://www.mobot.org/mobot/research/apweb/). Approximate branch lengths were added to the tree using the bladj function of Phylocom (Webb et al. 2008) and fixed node and tree root ages from Wikstrom et al. (2001). Analyses were conducted using the $\mathrm{R}$ program (ver. 3.1.0; $\mathrm{R}$ Development Core Team 2014). In addition, we tested the significance of correlations between growth rates and the two root traits using Spearman's rank correlation to account for outliers and nonnormal distributions. Data underlying the analyses are deposited in the Dryad Digital Repository: http://dx.doi.org/10.5061/dryad.49s82 (Lemmermeyer et al. 2015).

\section{Results \\ Growth Rates}

Both RGR and SGR varied among the 34 species. RGR after 10 weeks of growth ranged from 0.094 (Knautia arvensis) to 0.143 (Agrostis capillaris) $\mathrm{g} \mathrm{g}^{-1} \mathrm{day}^{-1}$ (table A1). SGR values calculated for the average plant size at week 6 ranged from 0.0004 ( $H$. perforatum) to 0.0750 (Rumex maritimus and Plantago major) $\mathrm{g} \mathrm{g}^{-1}$ day $^{-1}$ (table A1).

\section{Effects of Soil Treatments}

We expected species with faster growth to suffer more negative soil effects than slower growing species. Linear regression models of biotic soil effects including RGR or SGR were generally a better fit than simpler models (table 1). Both RGR and SGR varied negatively with biotic soil effects, with slower-growing species tending to be less negatively or even positively affected by the presence of soil biota (table 1; fig. 1). Removal of each species in turn revealed that parameter estimates for both RGR and SGR remained negative in all cases (table A4). A less steep relationship between RGR and biotic soil effects occurred in only nine cases when a species was removed compared with the model estimate including all species (table A4). The most influential species were Brachypodium sylvaticum (also an outlier, identified from a quantile-quantile normality plot), $H$. perforatum, K. arvensis, and Lathyrus pratensis; the latter two species showed net positive biotic soil effects (1.30 and 0.12), and their removal reduced the slope of the relationship (table A4). Removal of species led to a shallower relationship between SGR and biotic soil effects in 14 cases, and the most influential species were again $B$. sylvaticum, $K$. arvensis, and $L$. pratensis but also Plantago lanceolata (table A4). For conspecific soil effects, neither 
Table 1: Parameter estimates ( \pm standard error) and Akaike information criterion (AIC) for intercept models, intercept and biomass covariate models, and linear models including relative growth rate (RGR) or size-corrected growth rate (SGR) that explain (i) biotic soil effects or (ii) conspecific soil effects for 34 herbaceous plant species

\begin{tabular}{|c|c|c|c|c|}
\hline & \multicolumn{2}{|c|}{ Biotic soil effect } & \multicolumn{2}{|c|}{ Conspecific soil effect } \\
\hline & Estimate & AIC & Estimate & AIC \\
\hline Intercept & $-.042(.04)$ & 129.21 & $-.275(.089)$ & 73.43 \\
\hline Intercept & $-.701(.219)$ & 122.19 & $-.458(.100)$ & 69.75 \\
\hline Biomass (sum, g) & $-.546(.175)$ & & $-.216(.089)$ & \\
\hline Intercept & $2.117(.992)$ & 119.65 & $-.868(.734)$ & 70.68 \\
\hline Biomass (sum, g) & $-.139(.041)$ & & $-.056(.024)$ & \\
\hline RGR & $-18.388(8.738)$ & & $6.484(6.517)$ & \\
\hline Intercept & $.057(.060)$ & 118.56 & $-.253(.152)$ & 75.40 \\
\hline SGR & $-13.907(3.661)$ & & $-.466(2.805)$ & \\
\hline
\end{tabular}

of the linear models including RGR or SGR was distinguishable from simpler models according to the AIC, with no clear relationship between the growth rates and conspecific soil effects (table 1). The estimates for models explaining biotic and conspecific soil effects qualitatively differed little when phylogenetic information was included (table A5).

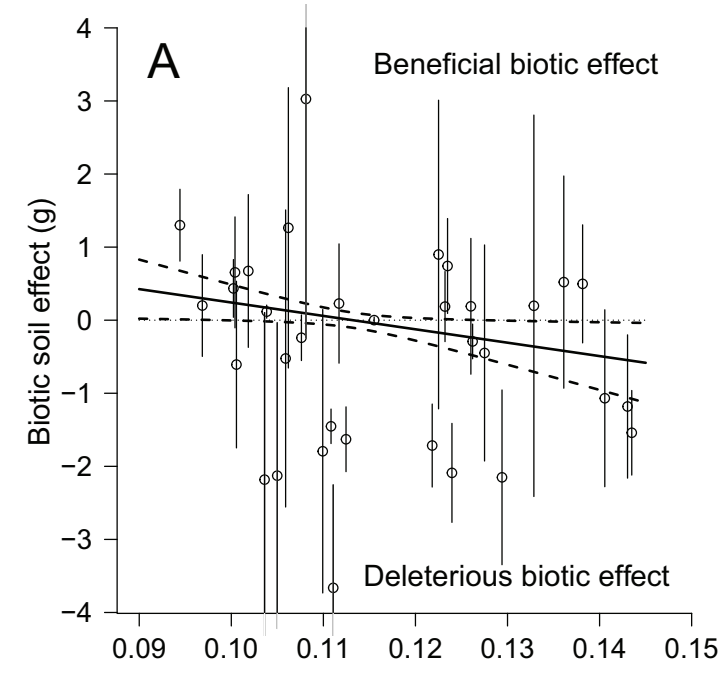

Relative growth rate (g/g/day)

\section{SRL and Root C:N Ratio}

We expected species' root traits to correlate with growth rates and, in turn, to be related to soil effects. SRL correlated positively with RGR, as predicted, but not with SGR (table 2). Root $\mathrm{C}: \mathrm{N}$ ratio correlated positively with $\mathrm{RGR}$ and SGR, but only significantly so for RGR (table 2). Phy-

Figure 1: Relationships between biotic soil effects on plant biomass and classical relative growth rate $(A)$ and size-corrected growth rate $(B)$. Negative soil effects indicate that plants had reduced biomass in unsterilized soils; positive soil effects indicate greater biomass in unsterilized soils. Error bars represent 95\% confidence intervals. The species with the largest weighting was Hypericum perforatum. Solid lines show fitted values from the models, and dashed lines show 95\% confidence intervals around the fitted lines. The horizontal line at $y=0$ represents a biotic soil effect of 0 (i.e., plant biomass in sterilized and unsterilized soil is equal). 
Table 2: Spearman's $\rho$ correlation coefficients and $P$ values (in parentheses) testing the correlations between relative growth rate (RGR) or size-corrected growth rate (SGR) and root C:N ratio or specific root length (SRL)

\begin{tabular}{llcc}
\hline & $n$ & RGR & SGR \\
\hline Root C: N: & & & \\
$\quad$ Raw & 31 & $.360(.047)$ & $.301(.099)$ \\
$\quad$ Contrasts & 31 & $.284(.129)$ & $.223(.235)$ \\
SRL $\left(\mathrm{cm} \mathrm{g}^{-1}\right):$ & & & \\
$\quad$ Raw & 33 & $.382(.029)$ & $-.195(.276)$ \\
Contrasts & 33 & $.499(.004)$ & $-.308(.087)$ \\
\hline
\end{tabular}

Note: Correlations were performed using raw root traits and growth rates per species as well as phylogenetically independent contrasts. The number of species $(n)$ considered in each correlation is also shown.

logenetically independent contrasts revealed a significant positive correlation between SRL and RGR but not between SRL and SGR (table 2). Contrasts of root C:N were not significantly correlated with RGR or SGR (table 2). Neither biotic nor conspecific soil effects showed a clear relationship with mean species' SRL, but biotic soil effects became more negative with increasing root $\mathrm{C}: \mathrm{N}$ (table A6), which also represented a better fit (AIC: 113.33) than the simpler model including only the biomass covariate (AIC: 118.97). However, this relationship was no longer negative after removal of the outlying and most influential species, B. sylvaticum (table A4).

\section{Discussion}

We expected faster-growing plant species to suffer more negatively from soil biota than slower-growing species and found some evidence in support of this expectation. Biotic soil effects varied negatively with RGR, switching from net beneficial to net deleterious effects of soil biota (fig. $1 \mathrm{~A}$ ). Similarly, biotic soil effects varied negatively with SGR, indicating that faster-growing species suffered more strongly from soil biota than slower-growing species (fig. 1B). For both measures of growth rate, the negative relationship was reduced when some of the most influential species were removed, suggesting that it may be driven by a subset of the species studied. Conspecific soil effects did not vary clearly with either RGR or SGR, whereas the effect of growing in conspecific soils relative to heterospecific soils on total biomass was significantly negative across all species (mean effect: $-0.275 \mathrm{~g}$; table 1 , intercept model). Therefore, some species-specific differences in soils must have been present.

Two caveats are warranted for the approaches used in our study. First, the soil sterilization approach used in plant-soil feedback studies can have unintended side effects, such as increasing nutrient availability (Trevors 1996), which may give the impression of greater plant biomass without soil biota. However, the volume of soil used to inoculate plants was less than $10 \%$ of the total volume of substrate, and the subsequent regular fertilization of plants would likely have overwhelmed any differences in nutrient availability caused by sterilization. Second, our estimates of RGR/SGR and soil effects may not be entirely representative of the species in general because of the relatively few genotypes sampled and the extra variation contributing to differences among species derived from sampling error. We cannot rule out that changes in sampling regime of source material might result in a differently shaped relationship. However, the same genotypes were used to estimate growth rates and soil effects, which should reduce the likelihood that a relationship between soil effects and growth rates is confounded by genotype differences.

What explains the relationships between effects of soil biota on plant growth and species growth rates? Plants engage in complex interactions with soil microbes via their roots, ranging from pathogenic (deleterious) through neutral to mutualistic (beneficial). Plant species differ in their associations with mycorrhizal fungi (Reinhart et al. 2012) and the direction of effect of these associations (Klironomos 2003). Moreover, mycorrhization varies with successional stage (Rasmann et al. 2011), and slower-growing species may depend more on mycorrhizal fungi than fastergrowing species (Reich 2014). Our results suggest some slower-growing species benefitted more from soil biota than faster-growing species (fig. 1). Such species (lower RGR) may benefit relatively more from soil mutualists than they suffer from pathogens, leading to net beneficial effects of soil biota, but this net benefit declines for species with faster growth rates until the effects of pathogens outweigh those of mutualists (net deleterious effects of soil biota). Interestingly, performance of the slower-growing Knautia arvensis is known to benefit consistently from association with arbuscular mycorrhizal fungi (Doubková et al. 2013). However, care is warranted in interpreting these linear relationships as general patterns, as they reflect responses by specific species, such as K. arvensis and Lathyrus pratensis, that grew slowly and benefitted from the presence of soil biota. Although models including growth rates explained sufficiently more variation than simple intercept and covariate models, much variation remain unexplained, and we cannot rule out the possibility that relationships could in fact be nonlinear. Determination of how generalizable the direction and shape of the relationship among angiosperms are will require assessment of more species.

If slower-growing species have a net benefit from soil mutualists and faster-growing species suffer a net deleterious effect from pathogens, then a key question is whether and how this difference is driven by differences in the roots 
of faster- and slower-growing species. We hypothesized that faster-growing species would have lower root $\mathrm{C}: \mathrm{N}$ ratio and greater SRL than slower-growing species. We found that SRL varied positively with RGR, indicating longer roots per unit mass for faster-growing species. However, $\mathrm{C}: \mathrm{N}$ ratio correlated positively with RGR (contrary to our hypothesis) and not with SGR. Root C: N varied negatively with biotic soil effects, which was also contrary to our prediction but was in line with its weak but positive correlation with growth rates (table 2). However, this significant relationship disappeared after accounting for the most influential species. Thus, the relationship between growth rates to soil biota effects may be linked to factors other than simple root traits.

Plant defense against soil pathogens will involve production of constitutive and induced defense compounds. Biochemical pathways involving salicylic acid are involved in plant defense against microbes (Vlot et al. 2009) but also appear to play a role in plant growth and development, as illustrated by transgenic salicylic-acid-depleted Arabidopsis plants exhibiting higher growth rates (Rivas-San Vicente and Piasencia 2011). Compounds such as salicylic acid could offer a direct mechanism for observed relationships between growth and defense both within and among species, but whether species with differing growth rates vary in expression of these compounds remains to be tested. Other attributes of roots and defense compounds could be linked to both plant growth rate and soil biota effects, but expression of these traits themselves can be mediated by soil organisms such as mycorrhizal fungi (Rasmann et al. 2011). As soil microbial communities are hyperdiverse, with many directly and indirectly interacting groups of species (Wardle et al. 2004), the relationship between belowground plant traits and the net effects of soil biota on plant growth is likely to be more complex than that for aboveground traits and natural enemies (Endara and Coley 2011).

To conclude, evidence partially supports a negative association between soil biota and plant growth rates. However, the considerable unexplained variation indicates that relationships may be more complex. Moreover, effects of soil biota appeared to be largely unrelated to the simple root traits SRL and root $\mathrm{C}: \mathrm{N}$ ratio, despite evidence that these traits were correlated with growth rates. Strong relationships between plant growth rates and defense from aboveground enemies may not be clearly reflected belowground because of the diversity and complexity of soil microbial communities, which involve multiple mutualistic and antagonistic interactions with plants. Further work could focus on disentangling the relationships between growth rates and effects of soil mutualists from those of pathogens to understand how these two groups might contribute to the relationships observed.

\section{Acknowledgments}

W.D. was funded by Deutsche Forschungsgemeinschaft (AZ DA 1502/1-1) and the University of Konstanz Young Scholar Fund (ZUK 52/2). Many thanks to R. Rohr for statistical advice and to T. Day and L. Harder for helpful comments on improving the manuscript. Thanks also to O. Ficht for horticultural assistance and K. MamanovaStift and K. Bär-Häge for technical laboratory assistance.

\section{Literature Cited}

Bever, J. D. 1994. Feedback between plants and their soil communities in an old field community. Ecology 75:1965-1977.

Brinkman, E. P., W. H. Van der Putten, E.-J. Bakker, and K. J. F. Verhoeven. 2010. Plant-soil feedback: experimental approaches, statistical analyses and ecological interpretations. Journal of Ecology 98:1063-1073.

Burnham, K. P., and D. R. Anderson. 2002. Model selection and multimodel inference: a practical information-theoretic approach. Springer, New York.

Chase, M. W., and J. L. Reveal. 2009. A phylogenetic classification of the land plants to accompany APG III. Botanical Journal of the Linnean Society 161:122-127.

Coley, P. D. 1988. Effects of plant-growth rate and leaf lifetime on the amount and type of anti-herbivore defense. Oecologia (Berlin) 74:531-536.

Coley, P. D., J. P. Bryant, and F. S. Chapin. 1985. Resource availability and plant antiherbivore defense. Science 230:895-899.

Comas, L. H., and D. M. Eissenstat. 2009. Patterns in root trait variation among 25 co-existing North American forest species. New Phytologist 182:919-928.

Doubková, P., P. Kohout, and R. Sudova. 2013. Soil nutritional status, not inoculum identity, primarily determines the effect of arbuscular mycorrhizal fungi on the growth of Knautia arvensis plants. Mycorrhiza 23:561-572.

Eissenstat, D. M. 1991. On the relationship between specific root length and the rate of root proliferation: a field study using Citrus rootstocks. New Phytologist 118:63-68.

Eissenstat, D. M., and R. D. Yanai. 1997. The ecology of root lifespan. Advances in Ecological Research 27:1-60.

Endara, M.-J., and P. D. Coley. 2011. The resource availability hypothesis revisited: a meta-analysis. Functional Ecology 25:389398.

Felsenstein, J. 1985. Phylogenies and the comparative method. American Naturalist 125:1-15.

Grime, J. P., and R. Hunt. 1975. Relative growth-rate: its ranges and adaptive significance in a local flora. Journal of Ecology 63:393-422.

Herms, D. A., and W. J. Mattson. 1992. The dilemma of plants: to grow or defend. Quarterly Review of Biology 67:283-335.

Jarosz, A. M., and A. L. Davelos. 1995. Effects of disease in wild plant populations and the evolution of pathogen aggressiveness. New Phytologist 129:371-387.

Kardol, P., G. B. De Deyn, E. Laliberté, P. Mariotte, and C. V. Hawkes. 2013. Biotic plant-soil feedbacks across temporal scales. Journal of Ecology 101:309-315.

Kempel, A., M. Schaedler, T. Chrobock, M. Fischer, and M. van Kleunen. 2011. Tradeoffs associated with constitutive and induced 
plant resistance against herbivory. Proceedings of the National Academy of Sciences of the USA 108:5685-5689.

Klironomos, J. N. 2003. Variation in plant response to native and exotic arbuscular mycorrhizal fungi. Ecology 84:2292-2301.

Kulmatiski, A., K. H. Beard, J. R. Stevens, and S. M. Cobbold. 2008. Plant-soil feedbacks: a meta-analytical review. Ecology Letters 11 980-992.

Lemmermeyer, S., L. Lörcher, M. van Kleunen, and W. Dawson 2015. Data from: Testing the plant growth-defense hypothesis belowground: do faster-growing herbaceous plant species suffer more negative effects from soil biota than slower-growing ones? American Naturalist, Dryad Digital Repository, http://dx.doi.org/10.5061 /dryad.49s82.

Lind, E., E. Borer, E. W. Seabloom, P. B. Adler, J. D. Bakker, D. Blumenthal, M. J. Crawley, et al. 2013. Life-history constraints in grassland plant species: a growth-defense trade-off is the norm. Ecology Letters 16:513-521.

Mangan, S. A., S. A. Schnitzer, E. A. Herre, K. M. L. Mack, M. C. Valencia, E. I. Sanchez, and J. D. Bever. 2010. Negative plant-soil feedback predicts tree-species relative abundance in a tropical forest. Nature 466:752-755.

Maron, J. L., J. Klironomos, L. Waller, and R. M. Callaway. 2014. Invasive plants escape from suppressive soil biota at regional scales. Journal of Ecology 102:19-27.

Packer, A., and K. Clay. 2000. Soil pathogens and spatial patterns of seedling mortality in a temperate tree. Nature 440:278-281.

Paine, C. E. T., T. R. Marthews, D. R. Vogt, D. Purves, M. Rees, A. Hector, and L. A. Turnbull. 2012. How to fit nonlinear plant growth models and calculate growth rates: an update for ecologists. Methods in Ecology and Evolution 3:245-256.

Pinheiro, J., D. Bates, D. Saikat, S. Deepayan, EISPACK authors, and R Development Core Team. 2014. 'nlme': linear and nonlinear mixed effects models. Version 3.1-118. Published October 7, 2014.

Rasmann, S., T. L. Bauerle, K. Poveda, and R. Vannette. 2011. Predicting root defense against herbivores during succession. Functional Ecology 25:368-379.

R Development Core Team. 2014. R: a language and environment for statistical computing. R Foundation for Statistical Computing, Vienna.

Reich, P. B. 2014. The worldwide "fast-slow" plant economics spectrum: a traits manifesto. Journal of Ecology 102:275-301.
Reinhart, K. O., G. W. T. Wilson, and M. J. Rinella. 2012. Predicting plant responses to mycorrhizae: integrating evolutionary history and plant traits. Ecology Letters 15:689-695.

Rivas-San Vicente, M., and J. Plasencia. 2011. Salicylic acid beyond defence: its role in plant growth and development. Journal of Experimental Botany 62:3321-3338.

Tjoelker, M. G., J. M. Craine, D. Wedin, P. B. Reich, and D. Tilman 2005. Linking leaf and root trait syndromes among 39 grassland and savannah species. New Phytologist 164:493-508.

Trevors, J. T. 1996. Sterilization and inhibition of microbial activity in soil. Journal of Microbiological Methods 26:53-59.

Turnbull, L. A., C. Paul-Victor, B. Schmid, and D. W. Purves. 2008. Growth rates, seed size, and physiology: do small-seeded species really grow faster? Ecology 89:1352-1363.

Turnbull, L. A., C. D. Philipson, D. W. Purves, R. L. Atkinson, J. Cunniff, A. Goodenough, Y. Hautier, et al. 2012. Plant growth rates and seed size: a re-evaluation. Ecology 93:1283-1289.

van der Putten, W. H., R. D. Bardgett, J. D. Bever, T. M. Bezemer, B. B. Casper, T. Fukami, P. Kardol, et al. 2013. Plant-soil feedbacks: the past, the present and future challenges. Journal of Ecology 101:265276.

van Zandt, P. A. 2007. Plant defense, growth, and habitat: a comparative assessment of constitutive and induced resistance. Ecology 88:1984-1993.

Vlot, A. C., D. A. Dempsey, and D. F. Klessig. 2009. Salicylic acid, a multifaceted hormone to combat disease. Annual Review of Phytopathology 47:177-206.

Wardle, D. A., R. D. Bardgett, J. N. Klironomos, H. Setala, W. H. van der Putten, and D. H. Wall. 2004. Ecological linkages between aboveground and belowground biota. Science 304:1629-1633.

Webb, C. O., D. D. Ackerly, and S. W. Kembel. 2008. Phylocom: software for the analysis of phylogenetic community structure and trait evolution. Bioinformatics 24:2098-2100.

Webb, C. O., and M. J. Donoghue. 2005. Phylomatic: tree assembly for applied phylogenetics. Molecular Ecology Notes 5:181-183.

Wikstrom, N., V. Savolainen, and M. W. Chase. 2001. Evolution of the angiosperms: calibrating the family tree. Proceedings of the Royal Society B: Biological Sciences 268:2211-2220.

Associate Editor: Lawrence D. Harder Editor: Troy Day 